\title{
Cardiogenic shock and complete heart block as a manifestation of fulminant Wegener's granulomatosis
}

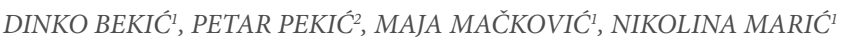 \\ ${ }^{1}$ Medical Intensive Care Unit, University Hospital Sveti Duh, Zagreb, Croatia \\ ${ }^{2}$ Department for Cardiovascular Disease, University Hospital Sveti Duh, Zagreb, Croatia
}

Corresponding author:

Dinko Bekić

Medical Intensive Care Unit, University Hospital Sveti Duh, Zagreb, Croatia

Phone: 01/3712-235

Fax: 01/3712-112

E-mail:dinkobekic@gmail.com

\section{ABSTRACT}

Wegener's vasculitis is an autoimmune disease affecting small and medium-sized blood vessels with a high mortality rate. It is a disease of usually subsidious course and nonspecific initial symptoms that are diagnostically misleading. Cardiac involvement is described most frequently in the form of supraventricular tachyarrhytmias, and rarely as a clinically significant myocardial infarction. This case demonstrates another facet of this disease presenting as full blown shock and respiratory insufficiency progressing to ARDS (acute respiratory distress syndrome) and multiorgan failure over the course of two weeks, with an unfortunately fatal outcome.

\section{INTRODUCTION}

Wegener's granulomatosis is a rare multisystem autoimmune disease of unknown etiology. It is characterised by necrotizing granulomatous inflammation and pauciimmune vasculitis in small- and medium-sized blood vessels. It predominantly affects the upper and lower respiratory tract and the kidneys, but can potentially affect any organ system. It is diagnosed by clinical features, immunology assays (anti-neutrophil cytoplasmic antibodies) and histology. Before the institution of effective therapy, the mean survival of adults with untreated GPA was only 5 months, with $82 \%$ of patients dying within the first year and $90 \%$ of patients dying within the second year. (1) Despite improvements with the use of corticosteroids, the mean survival time was increased to only 12.5 months. (1) Cardiac involvement occurs in 6 to $44 \%$ of cases. (2) The most frequent clinical manifestation are cardiac arrhythmias that are manifested as supraventricular tachyarrhythmias. We report a case of Wegener's vasculitis that manifested at admission as fulminant cardiogenic shock with multisystem failure and complete heart block.

Key words: Wegener's granulomatosis, vasculitis, cardiogenic shock

\section{CASE REPORT}

A 58-year-old female patient presented to the emergency department (ED) with syncope lasting for several seconds. Two weeks before presenting to the ED she had some haemoptysis and reported to the respiratory clinic. She complained of lethargy and fatigue during the last month long period. Pulmonary x-ray showed discrete, bilateral pulmonary infiltrates. The patient was diagnosed with bilateral pneumonia and oral cefpodoxim was prescribed. On the fifth day of antibiotic therapy she experienced nausea, retrosternal pain, vomited once and had syncope. The patient was admitted to the coronary care unit. Her medical history included: arterial hypertension, myocardial infarction in 2006, hyperlipdemia, and phlebothrombosis in 2014. Her medications included: warfarine, trimetazidine, amiodarone, isosorbide mononitrate, ramipril+hydrochlorothiazide, lacipidine. Physical examination showed pale skin, bilateral inspiratory crackles and a venous ulcer on her left lower leg. A 12lead electrocardiogram showed a new left bundle branch block. The bedside ECHO demonstrated slightly dilated left heart chambers with reduced systolic function of the left ventricle measuring 30\%, including inferior posterior wall hypokinesis. Right ventricle function was normal. Repeated laboratory tests showed an inflammatory response with leukocytosis of 15.91x109/ $\mu \mathrm{L}$ with a leftward shift, elevated C-reactive protein of $101 \mathrm{mg} / \mathrm{L}$, severe anaemia and elevated cardioselective enzymes. The findings were consistent with multi-organ dysfunction and metabolic acidosis. Chest $\mathrm{X}$-ray showed bilateral lung alveolar infiltrates (figure 1) that progressed rapidly over the course of a few hours and were consistent with adult respiratory distress syndrome (figure 2). In time, gradual hemodynamic deterioration required the combined use of vasopressors and inotropes, and continuous renal replacement therapy in parallel with an empirical broad spectrum antibiotic. The entire treatment lasted less than 48 hours. Although immunology assays weren't complete in such a short period, the overall clinical context suggested a possible autoimmune disease, so an ex iuvantibus pulse dose of corticosteroids was applied (methylprednisolone 1g). The respiratory and hemodynamic functions deteriorated rapidly, resulting in the patient being transferred to another centre for extracorporeal membrane oxygenation therapy (ECMO). However, the patient died before the therapy could be initiated. Afterwards, the elevated C-ANCA of 98.7 confirmed the diagnosis of Wegener's granulomatosis. 


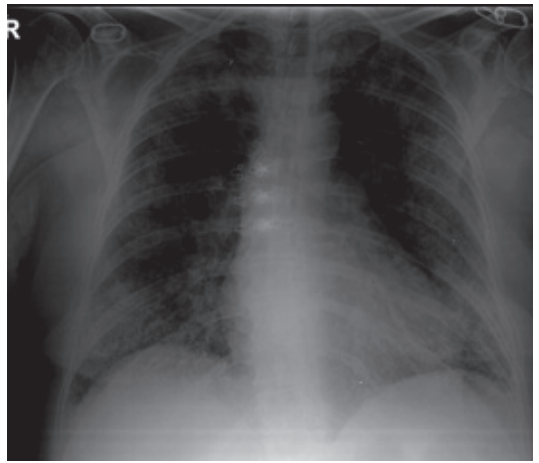

Figure 1. Bilateral, discrete pulmonary infiltrates

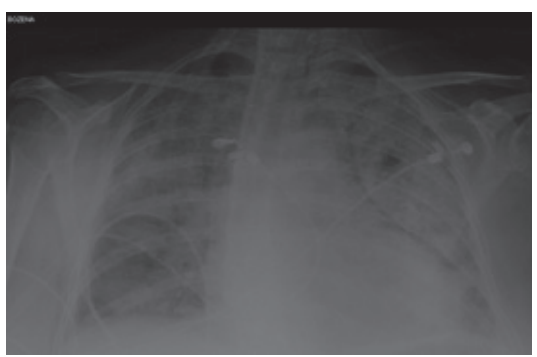

Figure 2. Bilateral pulmonary infiltrates - ARDS (Acute respiratory distress syndrome)

\section{DISCUSSION}

Wegener's patients typically present with constitutional, low intensity symptoms including fever, migratory arthralgias, malaise, anorexia and weight loss. Prodromal symptoms may last for weeks to months without evidence of specific organ involvement. (4) Significant cardiac complications occurring during the course of Wegener's granulomatosis are rare. (5) Cardiac involvement is rarely detected antemortem but includes pericarditis and coronary arteritis in $10-20 \%$ of cases. (5) Necrotizing vasculitis of the coronary vessels can result in a myocardial infarction or sudden death. (6) Clinically significant myocardial infarction is extremely rare in Wegener's granulomatosis. (6) A comprehensive review of 158 patients reported that $10(6 \%)$ developed pericarditis, eight of whom had typical clinical symptoms and signs. (6) An especially rare presentation is cardiogenic shock. Frequently, pulmonary involvement is more severe or fulminant. Our patient presented with cardiogenic shock and fulminant respiratory failure of unknown etiology and was refractory to all conservative treatments. The first working diagnosis on admission was acute coronary syndrome, but bilateral pulmonary infiltrates and haemoptysis rose suspicion for vasculitis as a primary diagnosis and intravenous corticosteroids were given in parallel with all other applied measures.
However, the clinical response was imperceptible. An ECMO procedure was indicated and the patient was transported to the national centre.

\section{CONCLUSION}

Antineutrophil cytoplasmic antibody (ANCA)-associated vasculitides include granulomatosis with polyangiitis (GPA), microscopic polyangiitis (MPA), the Churg-Strauss syndrome (CSS), and renal-limited vasculitis. All are associated with antineutrophil cytoplasmic antibodies (ANCA). (4) Clinical signs and symptoms are non-specific and diagnosis is a real challenge for physicians as it can be difficult to distinguish the signs and symptoms from other common diseases, such as infection or malignancy. The fulminant form of the disease is not common, but in such cases a rapid diagnostic evaluation and therapeutic strategy is of vital importance. In our case the course of the disease was abrupt and fulminant; it began with pulmonary infiltrates and haemoptysis that were misdiagnosed as pneumonia, and over the course of the next two weeks it progressed to ARDS and cardiogenic shock with multi-organ dysfunction syndrome. The patient was unfortunately in an irreversible stage of the disease and was refractory to all undertaken measures.

\section{REFERENCES}

1. Christopher L Tracy, MD, Patricia J Papadopoulos, MD.(Sep 25, 2014) Granulomatosis with Polyangiitis (Wegener Granulomatosis). Retrieved from: http://emedicine.medscape.com/article/332622-overview

2. R Coll Physicians Edinb. 2014;44(4):283-5. doi: 10.4997/JRCPE.2014.406. Granulomatosis with polyangiitis and constrictive pericarditis--a case report.

3. Horne AE1, Henriksen PA, Amft EN. Cardiac complications of wegener granulomatosis: A case report of complete heart block and review of the literature

4. Clinical manifestations and diagnosis of granulomatosis with polyangiitis and microscopic polyangiitis, Ronald J Falk, MD, Talmadge E King, Jr, MD;In: UpToDate, Post TW (Ed), UpToDate, Waltham, MA.

5. Silent myocardial infarction in wegener's granulomatosis t. m. lawson and b. d. williams Department of Rheumatology, University Hospital of Wales, Heath Park, Cardiff CF

6. Hoffman GS, Kerr GS, Leaitt RY et al. Wegener's granulomatosis: An analysis of 158 patients. Ann Intern Med 1992;116(6):488-98 